\title{
Application of the cell sheet technique in tissue engineering (Review)
}

\author{
GUANGNAN CHEN $^{1 *}$, YIYING QI $^{1 *}$, LIE NIU $^{2}$, TUOYU DI $^{1}$, \\ JINWEI ZHONG ${ }^{3}$, TINGTING FANG ${ }^{4}$ and WEIQI YAN ${ }^{1}$
}

\begin{abstract}
${ }^{1}$ Department of Orthopedic Surgery, The Second Affiliated Hospital, School of Medicine, Zhejiang University, Hangzhou, Zhejiang 310009; ${ }^{2}$ Department of Orthopedic Surgery, Dongping County People's Hospital, Taian, Shandong 271500; ${ }^{3}$ Department of Gastroenterology, The Second Affiliated Hospital, School of Medicine, Zhejiang University, Hangzhou, Zhejiang 310009; ${ }^{4}$ Key Laboratory of Carcinogenesis and Cancer Invasion, Fudan University, Ministry of Education, Liver Cancer Institute, Zhongshan Hospital, Xuhui, Shanghai 200032, P.R. China
\end{abstract}

Received April 2, 2015; Accepted September 7, 2015

DOI: 10.3892/br.2015.522

\begin{abstract}
The development and application of the tissue engineering technique has shown a significant potential in regenerative medicine. However, the limitations of conventional tissue engineering methods (cell suspensions, scaffolds and/or growth factors) restrict its application in certain fields. The novel cell sheet technique can overcome such disadvantages. Cultured cells can be harvested as intact sheets without the use of proteolytic enzymes, such as trypsin or dispase, which can result in cell damage and loss of differentiated phenotypes. The cell sheet is a complete layer, which contains extracellular matrix, ion channel, growth factor receptors, nexin and other important cell surface proteins. Mesenchymal stem cells (MSCs), which have the potential for multiple differentiation, are promising candidate seed cells for tissue engineering. The MSC sheet technique may have potential in the fields of regenerative medicine and tissue engineering in general. Additionally, induced pluripotent stem cell and embryonic stem cell-derived cell sheets have been proposed for tissue regeneration. Currently, the application of cell sheet for tissue reconstruction includes: Direct recipient sites implantation, superposition of cell sheets to construct three-dimensional structure for implantation, or cell sheet combined with scaffolds. The present review discusses the progress in cell sheet techniques, particularly stem cell sheet techniques, in tissue engineering.
\end{abstract}

Correspondence to: Professor Weiqi Yan, Department of Orthopedic Surgery, The Second Affiliated Hospital, School of Medicine, Zhejiang University, 88 Jie Fang Road, Hangzhou, Zhejiang 310009, P.R. China

E-mail: yanweiqizju@163.com

${ }^{*}$ Contributed equally

Key words: cell sheet, mesenchymal stem cells, extracellular matrix, implantation, tissue engineering

\section{Contents}

1. Introduction

2. Methods for harvesting cell sheets

3. Cell sheet techniques for tissue reconstruction

4. Applications of cell sheet techniques with non-mesenchymal stem cells

5. Mesenchymal stem cell sheets for tissue engineering

6. Tendon stem cell sheets for tendon tissue engineering

7. Other stem cell sheets and induced pluripotent stem cell sheets for tissue repair

8. Issues with cell sheet techniques

9. Conclusion

\section{Introduction}

The associated fields of regenerative medicine and tissue engineering have attracted increasing interest in the past 10-15 years, however, cell-based therapies have been clinically used for $<40$ years (1). Clinical products have also been yielded using tissue engineering with conventional methods of seeding cells into biodegradable scaffolds, and these products have applications in bone (2), cartilage (3) and blood vessels (4). Developing autologous tissue-engineered bladders was effective in treating patients with end-stage bladder disorders (5). Therefore, these results highlight the emergence of regenerative therapies using techniques that are superior to simple cell injection.

A strategy of 'cell sheet engineering', in which cultured cells are harvested as intact sheets along with their deposited extracellular matrix (ECM), was developed using temperature-responsive surfaces and can be applied in tissue engineering. By contrast, the use of temperature-responsive surfaces allows cultured cells to be harvested without the use of proteolytic enzymes, such as trypsin or dispase, as these can result in cell damage and loss of differentiated phenotypes $(6,7)$. Furthermore, as the ECM remains present on the basal surface of the cell sheets (8), they can be transplanted directly to tissue beds or even overlapped, creating three-dimensional (3D) 
tissue-like structures. This approach appears to exhibit numerous advantages over conventional regenerative therapies of cell injection and tissue reconstruction with biodegradable scaffolds. Frequently, there is a significant loss of cells, with only a small percentage of cells remaining at the target site when using single-cell suspension injections. Additionally, when the tissues are damaged, the injected cells may not be able to attach at sites where the host architecture is destroyed. By contrast, cell sheets with their deposited ECM, can attach to host tissues and even wound sites, covering the surface with minimal cell loss.

Mesenchymal stem cells (MSCs), which have the potential for multiple differentiation pathways, are promising candidate seed cells for tissue engineering. In general, the MSC sheet technique may have potential in the fields of regenerative medicine and tissue engineering. Additionally, induced pluripotent stem (iPS) cell and embryonic stem cell (ESC)-derived cell sheets have been proposed for tissue regeneration. The present review discusses the progress in cell sheet techniques, particularly stem cell sheet techniques, in tissue engineering.

\section{Methods for harvesting cell sheets}

Temperature-responsive culture dishes. Okano et al (9), developed an alternative cell sheet technique for regenerative therapies using temperature-responsive culture dishes. The temperature-responsive polymer poly( $N$-isopropylacrylamide) (PIPAAm) can undergo a distinct transition from hydrophobic to hydrophilic at its lower critical solution temperature of $32^{\circ} \mathrm{C}$. Thus, PIPAAm was immobilized covalently on ordinary tissue culture polystyrene (TCPS) surfaces at a nanometer-scale thickness, and subsequently cell adhesion and detachment can be controlled by simple temperature changes (10). On these surfaces, there were no differences in cell adherence, spread or proliferation of various types of cells compared with normal TCPS at $37^{\circ} \mathrm{C}$. Following this, cultured cells can be detached, in the form of a cell sheet, by reducing the incubation temperature, with the conversion of the coated PIPAAm from hydrophobic to hydrophilic. Varieties of cell sheets from different sources were harvested using the temperature-responsive culture dishes and the method of harvesting cell sheets is currently used widely in research. Additionally, overlapping cell sheets can be harvested and multilayered cell sheets can be constructed for tissue repair.

Polymerized human fibrin-coated dishes. Itabashi et al (11) cultured rat cardiomyocytes on polymerized human fibrin-coated dishes, prepared with fibrinogen monomers mixed with thrombin. As the fibrin had been digested by the intrinsic protease, the cell sheet could be readily dissociated intact from the polymerized fibrin layer. Cardiomyocytes cultured on these dishes formed a myocardial cell sheet within 4 days.

Vitamin C treatment. Wei et al (12) cultured periodontal ligament stem cells treated with vitamin $\mathrm{C}(\mathrm{Vc})$ at various concentrations. The cells form cell sheet structures at concentrations of $\mathrm{Vc}>20 \mu \mathrm{g} / \mathrm{ml}$, as $\mathrm{Vc}$ can increase cell matrix production. However, lower concentrations of $\mathrm{Vc}(0.0,5.0$ or $10.0 \mu \mathrm{g} / \mathrm{ml}$ ) did not result in the formation of sheets.

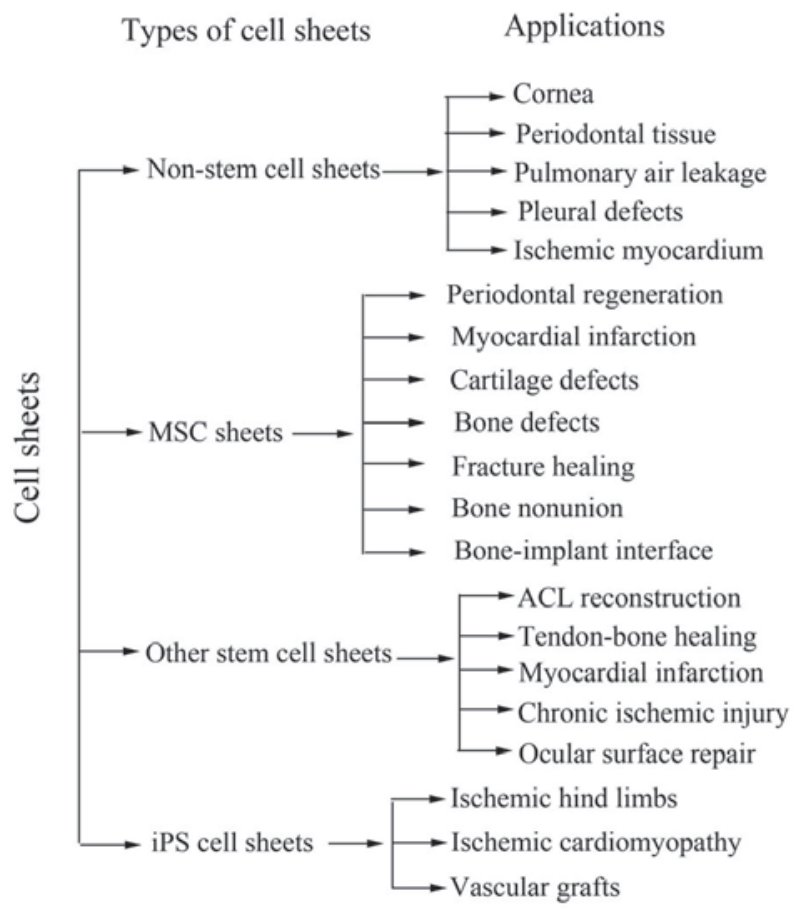

Figure 1. Application of different types of cell sheets. MSC, mesenchymal stem cells; iPS, induced pluripotent stem.

\section{Cell sheet techniques for tissue reconstruction}

Numerous studies using various types of cell sheet have been reported (Table I, Fig. 1). The methods for cell sheet preparation for tissue and organ reconstruction include single cell sheet implantation, cell sheets combined with scaffolds implantation and multilayer cell sheet implantation. Single cell sheet implantation has been used to repair periodontal ligament, skin, corneal epithelium and bladder epithelium. A cell sheet combined with scaffolds has been used to construct bone and cartilage. Multilayer cell sheet implantation has been used mainly for myocardium, smooth muscle, liver and other 3D tissues.

\section{Applications of cell sheet techniques with non-mesen- chymal stem cells}

Corneal repair. Corneal transplantation is commonly used for clinical corneal repair. However, the complications of transplantation rejection and donor limitations restrict its wider application. Tissue engineering methods can overcome such problems (13). First, autologous corneal epithelium cells are obtained using a proteolytic enzyme. Second, the corneal epithelium cells, combined with fibrin gel, collagen gel or amnion are transplanted onto the corneal stroma and held in place by sutures (14). However, the complications of inflammatory reactions at the receipt site, low corneal transparency and corneal opacity are common (14). With regards to these concerns, carrier-free constructs would be a better approach for ocular applications.

For the construction of corneal epithelial cell sheets, autologous limbal epithelial cells can be isolated and cultured on the temperature-responsive culture surfaces at $37^{\circ} \mathrm{C}$. Following 
Table I. In vivo studies using various cell sheets.

\begin{tabular}{|c|c|c|c|}
\hline Cell sheets & Applications & Models & (Refs.) \\
\hline \multicolumn{4}{|l|}{ Non-stem cell sheets } \\
\hline Epithelial cell sheets & Cornea & Humans & $(13)$ \\
\hline Oral mucosal epithelial cells & Cornea & Humans & $(14)$ \\
\hline Periodontal ligament cell sheets & Periodontal tissue & Dogs & $(15)$ \\
\hline Periodontal ligament cell sheets & Periodontal tissue & Canines & $(16)$ \\
\hline Periodontal ligament cell sheets & Periodontal tissue & Rats & $(17)$ \\
\hline Skin fibroblasts cell sheets & Pulmonary air leakage & Rabbits & $(18)$ \\
\hline Dermal fibroblast sheets & Pleural defects & Porcine & (19) \\
\hline Neonatal rat cardiomyocyte sheets & Ischemic myocardium & Rats & $(20)$ \\
\hline \multicolumn{4}{|l|}{ MSC sheets } \\
\hline Bone marrow-derived MSC sheets & Periodontal regeneration & Canine & $(21)$ \\
\hline Adipose tissue-derived MSC sheets & Myocardial infarction & Rats & $(22)$ \\
\hline Human adipose tissue-derived MSC sheets & Myocardial infarction & Nude rats & $(23)$ \\
\hline Bone marrow-derived MSC sheets & Cartilage defects & Rabbits & $(24)$ \\
\hline Bone marrow-derived MSC sheets & Bone defects & Rabbits & $(25-27)$ \\
\hline Bone marrow-derived MSC sheets & Fracture healing & Rats & $(28)$ \\
\hline Bone marrow-derived MSC sheets & Bone nonunion & Rats & $(29)$ \\
\hline Bone marrow-derived MSC sheets & Calvarial defects & Rabbits & $(30)$ \\
\hline Bone marrow-derived MSC sheets & Bone-implant interface & Rats & $(31)$ \\
\hline \multicolumn{4}{|l|}{ Other stem cell sheets } \\
\hline ACL-derived stem cell sheets & ACL reconstruction & Rats & $(32)$ \\
\hline Tendon-derived stem cell sheets & ACL reconstruction & Rats & $(33)$ \\
\hline Periosteal progenitor cell sheets & Tendon-bone healing & Rabbits & $(34)$ \\
\hline Muscle-derived stem cell sheets & Myocardial infarction & Mice & $(35)$ \\
\hline Cardiac stem cell sheets & Chronic ischemic injury & Pigs & $(36)$ \\
\hline Embryonic stem cell sheets & Ocular surface repair & Rabbits & $(37)$ \\
\hline \multicolumn{4}{|l|}{ iPS cell sheets } \\
\hline iPS-derived cell sheets & Ischemic hind limbs & Mice & $(38)$ \\
\hline iPS-derived cardiomyocytes-sheets & Ischemic cardiomyopathy & Pigs & (39) \\
\hline iPS-derived vascular cells sheets & Vascular grafts & Mice & $(40)$ \\
\hline
\end{tabular}

MSC, mesenchymal stem cells; iPS, induced pluripotent stem.

harvest, by reducing the temperature to $20^{\circ} \mathrm{C}$ for $30 \mathrm{~min}$, the epithelial cell sheets along with the deposited ECM can be readily manipulated and transplanted onto the host corneal stroma. After $5 \mathrm{~min}$, the transplanted cell sheet attaches to the corneal stroma without the requirement for sutures (15). Additionally, the cell sheet can cover the whole cornea and form a clear and smooth surface.

Tsai et al (16) implanted autologous epithelial cell sheets cultured on amniotic membrane to the denuded corneal surface of a damaged eye in 6 patients. Subsequently, 2-4 days later, complete reepithelialization of the corneal surface had occurred in all 6 eyes. The ocular surface was covered with corneal epithelium and the clarity of the cornea was improved 1 month after implantation. In 5 of the 6 eyes, the mean visual acuity improved from $20 / 112$ to $20 / 45$. The acuity improved from the ability to count fingers at $40 \mathrm{~cm}$ to $20 / 200$ for one patient with total opacification of the cornea from a chemical burn. Additionally, no patient had recurrent neovascularization or inflammation in the transplanted area during the 1.5 -year follow-up period.

In the study by Nishida et al (17), autologous oral mucosal epithelial cells were cultured on temperature-responsive cell-culture surfaces to form epithelial cell sheets, which were subsequently implanted directly on denuded corneal surfaces (1 eye for each patient) without sutures. The corneal surfaces were completely reepithelialized within 1 week in all 4 treated eyes. Corneal transparency was restored and postoperative visual acuity improved markedly in all 4 eyes. During a mean follow-up period of 14 months, all corneal surfaces remained transparent and no complications occurred.

Periodontal membrane repair. Conventional cells, scaffolds and growth factors have contributed significantly to improvements in the repair of periodontal tissue defects. However, due to the complexity of the regeneration of periodontal tissue and specificity of defects, the application of tissue engineering to 
periodontal tissue repair has been limited thus far. Cell sheet techniques have great potential in this area.

Flores et al (18) harvested osteogenic multilayered human periodontal ligament (PDL) cell sheets cultured in osteogenic medium using a temperature-responsive polymer dish and subcutaneously implanted the sheets into the back of immunodeficient rats. The majority of the dentin surfaces showed newly immature cementum-like tissue formation 6 weeks later and a periodontal ligament with perpendicular orientation was inserted into the newly deposited cementum-like tissue.

Akizuki et al (19) investigated periodontal healing in beagle dogs following application of a PDL cell sheet, which was prepared using temperature-responsive cell culture. Dehiscence defects were surgically created on the buccal surface of the mesial roots of bilateral mandibular first molars in each dog. In the experimental group, a PDL cell sheet with a reinforced hyaluronic acid carrier was applied to the defect. The hyaluronic acid carrier alone was applied to the contralateral side as a control. In the experimental group, periodontal tissue healing with bone, periodontal ligament and cementum formation was observed in 3 of 5 defects. In the control group, such periodontal tissue formation was only observed in one defect. Additionally, the formation of new cementum in the experimental group was significantly higher compared with the control group.

In the study by Iwata et al (20), three-layered PDL cell sheets were implanted, which were supported with woven polyglycolic acid, to dental root surfaces having three-wall periodontal defects in dogs. The bone defects were filled with porous $\beta$-tricalcium phosphate $(\beta$-TCP). Cell sheet transplantation regenerated new bone and cementum, which were connected with well-oriented collagen fibers, while only limited bone regeneration was observed in the control group.

In the study of Hasegawa et al (21), a human PDL cell sheet was transplanted into a mesial dehiscence model in athymic rats. Periodontal ligament-like tissues that included an acellular cementum-like layer and fibrils anchoring into this layer were identified in the cell sheet groups. This fibril anchoring strongly resembled native periodontal ligament fibers; no such regeneration was observed in the controls. These results suggest that PDL cell sheet transplantation may prove useful for periodontal regeneration in the clinical setting.

Pulmonary air leakage. Pulmonary air leakage commonly occurs in cardiothoracic surgeries, which reduces the quality of life for patients. Current methods include the use of various biological and synthetic sealants, which are ineffective in the closure of intraoperative air leaks. By contrast, the pulmonary air leakage cannot be permanently sealed, however, the sealants caused movement of the lung to be restricted (22). Bioengineered cell sheets can immediately and permanently seal air leaks in a dynamic manner, allowing for the extensive tissue contraction and expansion involved in respiration, without postoperative recurrence, when compared to previous materials, such as fibrin glue. The study by Kanzaki et al (23) used skin fibroblast cell sheets from rabbits as a novel lung air leak sealant and achieved an immediate and permanent lung air leak seal in the rabbit pulmonary air leakage model. Kanzaki et al (24) examined the effects of autologous dermal fibroblast sheets in the closure of pleural defects in a porcine model. The autologous dermal fibroblast sheets were attached directly to the lung surface, and provided an immediate seal against $<25 \mathrm{~cm} \mathrm{H}_{2} \mathrm{O}$ of airway pressure. The dermal fibroblast sheet remained present on the pleural surface 4 weeks after transplantation, providing permanent closure. The dermal fibroblast sheet was also responsive to changes in lung volume due to mechanical ventilation. No recurrence of air leaks was observed during the follow-up period. Thus, fibroblast cell sheets may be suitable for future clinical applications in repairing pulmonary air leakage.

Artificial vessel construction. Reconstructing a vascular network using layered cell sheets is a frequent task in 3D tissue engineering. Co-culture with endothelial cells (ECs) in the tissue was revealed to lead to in vitro pre-vascular network formation and promoted in vivo neovascularization following transplantation. Human umbilical vein endothelial cells (HUVECs), normal human dermal fibroblasts (NHDFs) and mixtures of the two cell types were harvested as intact cell sheets from temperature-responsive culture dishes at a low temperature $\left(<20^{\circ} \mathrm{C}\right)$ in the study by Asakawa et al (25). A single monoculture EC sheet was stacked with two NHDF sheets in different orders and three co-cultured cell sheets were layered with a cell sheet-collecting device. Pre-vascular networks, composed of HUVECs, were formed in all the triple-layer constructs, as revealed by morphological analyses. Confocal microscope observation showed that the pre-vascular networks formed tube structures similar to a native microvasculature.

Myocardial tissue engineering. Myocardial tissue engineering is one of the most promising treatments for patients suffering from severe heart failure. However, the problems of insufficient cell migration and cell loss with single-cell suspension injections and inflammatory reactions due to scaffold biodegradation with conventional tissue engineering remain to be elucidated. Use of layered myocardial cell sheets to construct 3D functional tissues with no artificial scaffold is promising in myocardial tissue engineering. Shimizu et al (26) reported that electrical communications could be established between layered cardiomyocyte sheets, resulting in simultaneously beating 3D myocardial tissues. Additionally, layered cardiomyocyte sheets in vivo showed a long survival, macroscopic pulsation and structures characteristic of native heart tissue.

Shimizu et al (27) harvested triple-layer neonatal rat cardiomyocyte sheets and repeatedly implanted them into the subcutaneous tissue of nude rats at 1-, 2- or 3-day intervals. The two overlaid grafts completely synchronized and the whole tissue survived without necrosis in the 1- and 2-day interval cases. Multistep transplantation also created an 1-mm thick myocardium with a well-organized microvascular network. Furthermore, complete graft perfusion via the vessels was revealed following the fabrication of the functional multilayer graft over a surgically connectable artery and vein. Miyagawa et al (28) identified that neonatal rat cardiomyocyte sheets were integrated with an impaired myocardium and improved the cardiac performance in a model of ischemic myocardium in rats. The cardiomyocyte sheets became attached to the infarcted myocardium, revealing angiogenesis, and appeared to be a homogeneous tissue in the myocardium. 
Echocardiography demonstrated that cardiac performance was significantly ameliorated following the implantation. Electrophysiological experiments revealed a QRS complex with one peak in the treated scar area in the sheet group, and two peaks in the other groups, indicating branch block. Furthermore, the threshold for pacing of the recipient heart was lower in the sheet group compared to the other groups. Such cell sheet integration methods may provide a possible solution for the clinical repair of various damaged organs, including impaired myocardium.

\section{Mesenchymal stem cell sheets for tissue engineering}

MCSs are used widely in the field of tissue engineering and have been demonstrated as a promising candidate cell source. MSCs can form in the structure of a sheet, providing large numbers of cells and extracellular matrix, and may be useful for tissue repair and regeneration in diverse fields.

Periodontal regeneration. Tsumanuma et al (29) performed cell sheet transplantation using three types of mesenchymal tissue-derived cells (periodontal ligament, alveolar periosteum and bone marrow) to compare differences between cell sources in a canine severe defect (one-wall intrabony defect) model. Three-layered cell sheets from each cell source, supported with woven polyglycolic acid, were transplanted autologously to the denuded root surface. One-wall intrabony defects were filled with a mixture of $\beta$-TCP and collagen. Periodontal regeneration with newly formed cementum and well-oriented PDL fibers and alveolar bone regeneration was identified in all the groups 8 weeks after transplantation. The highest amount of bone and periodontal regeneration was revealed in the PDL sheet group.

MSC sheets for cardiac tissue regeneration. MSCs have been used commonly for cardiac tissue repair in animal models and using cell suspension systems clinically. Due to the disadvantages of MSC suspensions, MSC sheets have a potential in cardiac tissue regeneration. Miyahara et al (30) cultured adipose tissue-derived MSC (ADMSCs) sheets using temperature-responsive culture dishes and implanted them onto scarred myocardium in rats. The engrafted sheet grew gradually to form a thick stratum that included newly formed vessels, undifferentiated cells and a few cardiomyocytes. The MSC sheet reversed wall thinning in the scar area and improved cardiac function in rats with myocardial infarction. Okura et al (31) induced human ADMSCs (hADMSCs) to differentiate into cardiomyoblast-like cells (CLCs) with dimethylsulfoxide, which were used to treat myocardial infarction in a nude rat model. The beneficial effects on cardiac function of hADMSC sheets and CLCs sheets were compared. Echocardiography revealed a significant short-term improvement in cardiac function in the patch-transplanted groups. However, rescue and maintenance of cardiac function was only apparent in the hCLC patch-transplanted group as revealed by long-term follow-up, and not in the non-committed hADMSC patch-transplanted animals. The hCLCs, and not the hADMSCs, were engrafted into the scarred myocardium and differentiated into human cardiac troponin I-positive cells. These were therefore regarded as cardiomyocytes.
Transplantation of the hCLC patches resulted in the recovery of cardiac function and improvements in the long-term survival rate. Thus, transplantation of mesenchymal stem cell sheets or differentiated cardiomyoblast-like cell sheets may provide a new therapeutic strategy for cardiac tissue regeneration.

MSC sheets for cartilage integration and repair. Injured cartilage can barely repair itself automatically due to its limited self-healing capacity, often leading to further degeneration when untreated. Tissue engineering has been demonstrated as a promising approach for restoring cartilage defects. To achieve enduring healing and biomechanical competence of cartilage, cartilage integration is important. MSC-loaded scaffolds have been implanted into cartilage defects directly. However, there is a clear discontinuity between the implant and host cartilage, creating a focus for failure. Additionally, integrative cartilage repair may be hindered by a lack of matrix-producing cells in the interface area. Mesenchymal stem cell sheets can provide large numbers of cells and extracellular matrix, which may provide continuity between the implant and host cartilage, thus improving integrative cartilage repair. Additionally, adhesion molecules on cell surfaces and cell-cell interactions remain intact.

Qi et al (32) investigated the effects of incorporating the properties of MSC sheets into MSC-loaded bilayer poly-(lactic-co-glycolic acid) (PLGA) scaffolds to improve the integration and repair of cartilage defects in a rabbit model. At 6 and 12 weeks after implantation, the MSC sheet-encapsulated PLGA/MCSs group showed significantly more hyaline cartilage and higher histological scores compared with the other groups. Additionally, the MSC sheet-encapsulated PLGA/MCSs group showed the optimal integration between the repaired cartilage and surrounding normal cartilage and subchondral bone. Furthermore, Qi and Yan (33) hypothesized that the MSC sheet-encapsulated cartilage debris may promote cartilage repair efficiently in patients with OA. Chondrocytes can be obtained and cultured from small cartilage debris in vitro. Therefore, the chondrocytes may grow from the debris in a cartilage defect and improve cartilage regeneration. MSC sheets provide large numbers of cells with ECM and proteins for cartilage regeneration and integration, and may have a certain role in the periosteum. In addition, cell sheet/cartilage debris constructs can be readily shaped based on the size and shape of the cartilage defect. MSC sheets may have a potential in cartilage integration and regeneration, even in patients with OA.

MSC sheets for bone regeneration. MSCs are multipotent cells that have positive roles in bone regeneration. In a previous study, MSCs combined with various materials were shown to regenerate bone defects using cell suspension systems (34). However, the adhesion rate of MSCs is low due to the low surface area to volume ratio of scaffolds. The cell sheet technique can achieve highly efficient cell delivery (35). Additionally, the adhesion molecules on the cell surface and cell-cell interactions remain intact. Furthermore, osteoblasts attached to the mineralized layered cell sheet may mimic the in vivo deposition of bone matrix.

Ouyang et al (36) investigated the feasibility of fabricating sheets of MSCs to revitalize cryopreserved grafts. Human 
MSC sheets assembled on demineralized bone grafts or frozen tendon grafts by a wrapping technique were cultured for 3 weeks. When assembled with demineralized bone matrix, the MSC sheet was similar to the in situ periosteum and could differentiate into the osteochondral lineage.

When assembled with a frozen tendon graft, the MSC sheet was incorporated within the tissue sheath (peritenon) around the tendon and adopted the characteristic spindle-shaped morphology of tenocyte-like cells. This study further verified the potential of in situ-specific differentiation of MSC sheet cells.

In the study by Gao et al (37), multilayered bone marrow stromal cell (BMSC) sheets were assembled with tubular coral scaffolds for long bone regeneration (5-mm diameter and $1.5-\mathrm{mm}$ wall thickness) in rabbits. Cortical bone with a similar shape and structure to native bone was formed. Furthermore, the new bone formation was in a similar manner to endochondral osteogenesis, with woven bone matrix subsequently maturing into fully mineralized compact bone. Zhou et al (38) used multilayered porcine BMSC sheets, assembled with fully interconnected polycaprolactone-calcium phosphate scaffolds, to engineer structural and functional bone grafts. Two sets of cell-sheet-scaffold/cell constructs were transplanted under the skin of nude rats in vivo. The first constructs $(5 \times 5 \times 4 \mathrm{~mm})$ were assembled with BMSC sheets and cultured for 8 weeks prior to implantation. The second set of constructs (10x10x4 mm) was implanted immediately following the assembly with BMSC sheets. In the two groups, neocortical and well-vascularized cancellous bone was formed within the constructs, with $\leq 40 \%$ bone volume. The neobone tissue formed from the pool of seeded BMSCs and the bone formation predominantly followed an endochondral pathway, with woven bone matrix subsequently maturing into fully mineralized compact bone, exhibiting the histological markers of native bone. In the study of Zou et al (39), assembled BMSC sheet/allografts structures were implanted subcutaneously into nude mice and into the segmental radius defect of rabbits. Following implantation, the MSC sheet accelerated the repopulation of the bone graft in nude mice. In addition, the MSC sheet induced thicker cortical bone formation and more efficient graft-to-bone end fusion at segmental bone defects in rabbits.

Qi et al (40) implanted human bone morphogenetic protein-2 (rhBMP-2)-loaded calcium sulfate (CS)/MSC sheets to repair ulnar segmental defects in rabbits. The defects treated with MSC sheet/rhBMP-2-loaded CS showed significantly higher scores, by X-ray analysis, and more bone formation, determined by histology and microcomputed tomography, compared to the other groups at 4 and 8 weeks after implantation $(\mathrm{P}<0.05)$. The MSC sheet had significant roles in bone regeneration. Qi et al (41) also investigated the effects of simvastatin that was applied locally from CS/MSC sheets on fracture healing in a rat tibia model. At 8 weeks, complete bone union was obtained in the CS/simvastatin/MSC sheet group. By contrast, newly regenerated bone tissue only partially bridged the gap or non-union in the other groups.

Nakamura et al (42) harvested rat osteogenic BMSC sheets and cell sheets were transplanted onto fractured sites without a scaffold in a femur nonunion model in rats. X-ray photographs and histological sections showed callus formation around the fracture site in the cell sheet-transplanted group (sheet group).
Bone union was obtained in the sheet group at 8 weeks. The results of a pullout evaluation in the vertical direction of the femur in the sheet group were significantly improved compared to those of the control group. Analysis of the origin of de novo-formed bone using the Sry gene, a marker of the donor cells, showed that the transplanted cells without scaffolds could survive and differentiate into osteogenic lineage cells in vivo. Ma et al (43) investigated the capacity of BMSC sheets to heal critical-size rabbit calvarial defects. At 6 and 12 weeks after implantation, the defects treated with demineralized bone matrix/cell sheet showed the greatest bone formation. Such MSC sheet and tissue engineering strategies offer therapeutic opportunities for promoting bone defect repair in clinical situations.

MSC sheet at the interface between bone and implant. Due to the high morbidity associated with artificial joint loosening, promoting bonding behavior and increasing bone ingrowth between bone and implant is important for the stability of the joint. A novel MSC sheet-wrapped implant can promote bone bonding at the bone-implant interface by providing cells and ECM.

In the study of Zhou et al (44), multilayered rabbit BMSC sheets were assembled with two types of implant (surface-modified titanium and zirconia) to construct an MSC sheet-implant complex. After culturing in osteogenic medium for 2 weeks, two types of cell sheet-implant complex were transplanted under the skin of SCID mice for 8 weeks. For the MSC sheet-titanium implant complex, new bone tissue that formed around the implants followed a predominantly endochondral pathway, exhibiting histological markers of native bone. Intramembranous ossification appeared to occur on the surface of the zirconia implant, as observed with typical osteocytes embedded in a dense matrix and accompanied by microvessels and marrow cavities in the MSC sheet-zirconia implant complex. Similarly, Yu et al (45) harvested MSC sheets and wrapped them around titanium implants to construct complexes, followed by culturing in an osteogenic medium. The effect of an MSC sheet-implant complex in the right tibia of a diabetic rat model was investigated. The MSC sheet-implant complex showed a significantly higher bone volume ratio and trabecular thickness $(\mathrm{P}<0.05)$ and significantly less trabecular separation $(\mathrm{P}<0.05)$ compared to the titanium implants in the diabetic rats 4 and 8 weeks later. Additionally, the amount of new bone tissue forming around the MSC-implant complexes was higher in comparison to with the titanium implants.

Cell sheet engineering techniques in conjunction with routine implant materials can generate MSC-implants possessing osteogenic and vascularization abilities. Therefore, the MSC-implant complexes provide a novel tissue engineering approach that promotes osseous healing and may be useful in the treatment of patients with diabetes.

\section{Tendon stem cell sheets for tendon tissue engineering}

In addition to MSCs, other adult stem cells can be harvested and cultured to form sheet structures, which can then be applied in specific fields, such as ACL reconstruction. In ACL reconstruction, tendon-bone tunnel healing is crucial for long-term success. Use of a cell sheet to improve tendon-bone healing has 
been investigated. Mifune et al (46) developed an ACL-derived $\mathrm{CD}^{+}{ }^{+}$stem cell sheet-wrapped tendon graft to improve recovery following ACL reconstruction in a rat model. The results demonstrated that the cells derived from the cell sheets incorporated within the bone tunnel site and grafted tendon and that healing of the bone-tendon junction and the grafted tendon was significantly enhanced. Similarly, to promote early graft healing following ACL reconstruction in a rat model, Lui et al (47) wrapped a tendon-derived stem cell (TDSC) sheet around the ACL graft. At weeks 2, 6 and 12 after reconstruction, the TDSC group showed a time-dependent increase in tunnel bone mineral density and bone volume/total volume at the epiphyseal region of the tibial tunnel. Semiquantitative image analysis showed improved graft osteointegration and higher intra-articular graft integrity with lower cellularity and vascularity, better cell alignment, and higher collagen birefringence. Additionally, the ultimate load and stiffness were significantly higher in the TDSC group. Chang et al (48) investigated tendon-bone healing in an extra-articular bone tunnel model in rabbits using periosteal progenitor cell (PPC) sheets. PPC sheets were used as a periosteum to wrap around hamstring tendon grafts; they were pulled into a $3-\mathrm{mm}$ diameter bone tunnel of the tibia. PPC sheets enhanced collagen and glycosaminoglycans deposition with fibrocartilage formation in the tendon-bone junction. Matured fibrocartilage and dense collagen fiber were formed at the tendon-bone interface. Bioengineered PPC or ACL or tendon stem cell sheets may offer a novel and feasible therapeutic strategy to enhance ACL recovery and tendon-bone junction healing.

\section{Other stem cell sheets and induced pluripotent stem cell sheets for tissue repair}

Other types of stem cell can be obtained from adult tissues and can form cell sheets. For myocardial infarction, cardiac stem cell sheets and muscle stem cell sheets are promising. Sekiya et al (49) transplanted a muscle-derived stem cell (MDSC) sheet in a mouse model of chronic myocardial infarction. MDSC sheet-implanted mice exhibited a reduction in left ventricle (LV) dilation and sustained LV contraction compared with the other groups. The MDSC sheet formed aligned myotubes and yielded better functional recovery of chronic infarcted myocardium, with no significant arrhythmic events.

The transplantation of stem cell sheets is also a promising therapeutic strategy for ischemic cardiomyopathy. However, potential ischemia in the transplanted area remains a problem. Endothelial progenitor cells (EPCs) can induce angiogenesis in the injected area. Thus, in the study of Kamata et al (50) a cardiac stem cell (CSC) sheet, combined with intramyocardial EPC injection, was assessed in a porcine chronic ischemic injury model, generated by placing an ameroid constrictor around the left coronary artery for 4 weeks. CSC sheet implantation with PC injection yielded significant functional recovery in the ischemic epicardium with increasing capillary number and reduced cardiac fibrosis in the ischemic epicardium or endocardium.

ESCs are considered to be the best potential candidate seed cells for induced differentiation for all tissue engineering and regeneration without the limitations of donor site morbidity for adult stem cells. Matsuura et al (51) created sheets of mouse embryonic stem (ES) cell-derived cardiomyocytes following expansion in 3D stirred suspension cultures and co-culture with cardiac fibroblasts. The cardiomyocytes in the cell sheets beat spontaneously and synchronously. Such ES-derived cardiac cell sheets may be a promising tool for the development of bioengineered myocardium. Zhang et al (52) used ESC sheets for ocular surface reconstruction in rabbits with limbal stem cell deficiency using the centrifugation method. The cell sheet transplant facilitated reconstruction of the ocular surface in $75 \%$ of the treated animals. The ES cells differentiated into corneal epithelial cells when in direct contact with the stroma and thus can serve as a cell source for corneal tissue engineering. However, several key problems are associated with ESCs, such as ethical issues, immunological rejection and the risk of teratoma formation (53), which require resolving prior to their more extensive application.

Compared with cardiac stem cell sheets, muscle stem cell sheets and ESCs, the successful induction and isolation of iPS cells provide new hope. iPS cells can be created from a patient's own somatic cells and also have the potential to differentiate into multiple types, leading to a genuinely personalized therapy. The iPS cell-differentiated cell sheets, following induction, have been investigated widely in tissue regeneration. Kito et al (54) used mouse iPS cell-derived Flk-1(+) cell sheets for repairing ischemic hind limbs in a nude mice model. Following implantation, Doppler blood flow and capillary density analyses showed that revascularization of ischemic hind limbs was accelerated and the Flk-1(+) cell sheet also increased the expression of VEGF and bFGF in ischemic tissue. Kawamura et al (55) used human induced pluripotent stem cell-derived cardiomyocyte (hiPS-CM) sheet transplantation for the treatment of ischemic cardiomyopathy in a porcine model, which was induced by ameroid constriction of the left anterior descending coronary artery. hiPS-CMs could be detected 8 weeks after transplantation. Therefore, the cell sheet transplantation significantly improved the cardiac performance and attenuated left ventricular remodeling. Additionally, no teratoma formation was observed in animals that received hiPS-CM sheets. However, potential ischemia may limit cell sheet survival. The omentum, which is known to have a rich vasculature, is expected to be the blood supply source. Kawamura et al (56) demonstrated that the omentum flap enhanced the survival of hiPS-CMs following transplantation via increased angiogenesis. The combination of hiPS-CMs and the omentum flap may be a promising technique for the development of tissue-engineered vascular-rich new myocardium in vivo.

Differentiated iPS cells sheets have also been examined for construction of tissue-engineered vascular grafts. iPS cell-derived vascular cell sheets wrapped on a scaffold composed of polyglycolic acid-poly-1-lactide and poly(1-lactide-co-epsilon-caprolactone) with a diameter of $0.8 \mathrm{~mm}$ were implanted as interposition grafts in the inferior vena cava of severe combined immunodeficiency/beige female mice. Endothelialization and an inner layer with smooth muscle actin- and calponin-positive cells were observed via histological evaluation at 10 weeks (57).

\section{Issues with cell sheet techniques}

Cell sheet techniques can overcome specific disadvantages of conventional tissue engineering methods. However, certain 
problems remain. A cell sheet is thin, so therefore, to generate thicker cell sheets, they are often overlapped, layer by layer. However, necrosis can occur in the middle of multilayered cell sheets due to poor nutrition or hypoxia. Additionally, potential ischemia in vivo may limit cell sheet survival. A previous study showed that hypoxia pretreatment could increase the survival rate of implanted MSCs and promote angiogenesis in vivo (58). Therefore, hypoxia-pretreated cell sheets may have better survival rates and improved therapeutic efficacy in vivo. Furthermore, the study of cell sheets derived from ESCs and iPS cells remains in the early stages. Wider applications of ESC- or iPS-derived cell sheets remain to be investigated.

\section{Conclusion}

Cell sheet techniques have been used widely for tissue regeneration and repair. Cell sheet tissue engineering, particularly MSC sheet tissue engineering, is becoming an important tool for regenerative medicine. Hypoxia-pretreated and angiogenesis MSC sheets combined with gene transfer and/or growth factors have a significant potential in tissue regeneration. In the future, ESC- or iPS-derived cell sheets may also be useful subsequent to overcoming several challenges.

\section{Acknowledgements}

The project was supported by the Natural Science Foundation of Zhejiang Province (grant no. LQ14H060001) and the Natural Science Foundation of China (grant no. 81401779).

\section{References}

1. Thomas E, Storb R, Clift RA, Fefer A, Johnson FL, Neiman PE, Lerner KG, Glucksberg H and Buckner CD: Bone-marrow transplantation (first of two parts). N Engl J Med 292: 832-843, 1975.

2. Vacanti CA, Bonassar LJ, Vacanti MP and Shufflebarger J: Replacement of an avulsed phalanx with tissue-engineered bone. N Engl J Med 344: 1511-1514, 2001.

3. Qi Y, Zhao T, Xu K, Dai T and Yan W: The restoration of full-thickness cartilage defects with mesenchymal stem cells (MSCs) loaded and cross-linked bilayer collagen scaffolds on rabbit model. Mol Biol Rep 39: 1231-1237, 2012.

4. Poh M, Boyer M, Solan A, Dahl SL, Pedrotty D, Banik SS, McKee JA, Klinger RY, Counter CM and Niklason LE: Blood vessels engineered from human cells. Lancet 365: 2122-2124, 2005.

5. Atala A, Bauer SB, Soker S, Yoo JJ and Retik AB: Tissue-engineered autologous bladders for patients needing cystoplasty. Lancet 367: 1241-1246, 2006.

6. Kushida A, Yamato M, Isoi Y, Kikuchi A and Okano T: A noninvasive transfer system for polarized renal tubule epithelial cell sheets using temperature-responsive culture dishes. Eur Cell Mater 10: 23-30, 2005.

7. Kushida A, Yamato M, Kikuchi A and Okano T: Two-dimensional manipulation of differentiated Madin-Darby canine kidney (MDCK) cell sheets: The noninvasive harvest from temperature-responsive culture dishes and transfer to other surfaces. J Biomed Mater Res 54: 37-46, 2001.

8. Kushida A, Yamato M, Konno C, Kikuchi A, Sakurai Y and Okano T: Decrease in culture temperature releases monolayer endothelial cell sheets together with deposited fibronectin matrix from temperature-responsive culture surfaces. J Biomed Mater Res 45: 355-362, 1999.

9. Okano T, Yamada N, Sakai H and Sakurai Y: A novel recovery system for cultured cells using plasma-treated polystyrene dishes grafted with poly(N-isopropylacrylamide). J Biomed Mater Res 27: 1243-1251, 1993.

10. Okano T, Yamada N, Okuhara M, Sakai H and Sakurai Y: Mechanism of cell detachment from temperature-modulated hydrophilic-hydrophobic polymer surfaces. Biomaterials 16: 297-303, 1995.
11. Itabashi Y, Miyoshi S, Kawaguchi H, Yuasa S, Tanimoto K, Furuta A, Shimizu T, Okano T, Fukuda K and Ogawa S: A new method for manufacturing cardiac cell sheets using fibrin-coated dishes and its electrophysiological studies by optical mapping. Artif Organs 29: 95-103, 2005.

12. Wei F, Qu C, Song T, Ding G, Fan Z, Liu D, Liu Y, Zhang C, Shi $\mathrm{S}$ and Wang S: Vitamin $\mathrm{C}$ treatment promotes mesenchymal stem cell sheet formation and tissue regeneration by elevating telomerase activity. J Cell Physiol 227: 3216-3224, 2012.

13. Nishida K: Tissue engineering of the cornea. Cornea 22 (Suppl 7): S28-S34, 2003

14. Yang J, Yamato M, Nishida K, Ohki T, Kanzaki M, Sekine H, Shimizu T and Okano T: Cell delivery in regenerative medicine: The cell sheet engineering approach. J Control Release 116: 193-203, 2006.

15. Nishida K, Yamato M, Hayashida Y, Watanabe K, Maeda N, Watanabe H, Yamamoto K, Nagai S, Kikuchi A, Tano Y, et al: Functional bioengineered corneal epithelial sheet grafts from corneal stem cells expanded ex vivo on a temperature-responsive cell culture surface. Transplantation 77: 379-385, 2004.

16. Tsai RJ,Li LM and Chen JK: Reconstruction of damaged corneas by transplantation of autologous limbal epithelial cells. N Engl J Med 343: 86-93, 2000.

17. Nishida K, Yamato M, Hayashida Y, Watanabe K, Yamamoto K, Adachi E, Nagai S, Kikuchi A, Maeda N, Watanabe H, et al: Corneal reconstruction with tissue-engineered cell sheets composed of autologous oral mucosal epithelium. N Engl J Med 351: 1187-1196, 2004.

18. Flores MG, Hasegawa M, Yamato M, Takagi R, Okano T and Ishikawa I: Cementum-periodontal ligament complex regeneration using the cell sheet technique. J Periodontal Res 43: 364-371, 2008

19. Akizuki T, Oda S, Komaki M, Tsuchioka H, Kawakatsu N, Kikuchi A, Yamato M, Okano T and Ishikawa I: Application of periodontal ligament cell sheet for periodontal regeneration: A pilot study in beagle dogs. J Periodontal Res 40: 245-251, 2005.

20. Iwata T, Yamato M, Tsuchioka H, Takagi R, Mukobata S, Washio K, Okano T and Ishikawa I: Periodontal regeneration with multi-layered periodontal ligament-derived cell sheets in a canine model. Biomaterials 30: 2716-2723, 2009.

21. Hasegawa M, Yamato M, Kikuchi A, Okano T and Ishikawa I: Human periodontal ligament cell sheets can regenerate periodontal ligament tissue in an athymic rat model. Tissue Eng 11: 469-478, 2005

22. Fabian T, Federico JA and Ponn RB: Fibrin glue in pulmonary resection: A prospective, randomized, blinded study. Ann Thorac Surg 75: 1587-1592, 2003.

23. Kanzaki M, Yamato M, Yang J, Sekine H, Kohno C, Takagi R, Hatakeyama H, Isaka T, Okano T and Onuki T: Dynamic sealing of lung air leaks by the transplantation of tissue engineered cell sheets. Biomaterials 28: 4294-4302, 2007.

24. Kanzaki M, Yamato M, Yang J, Sekine H, Takagi R, Isaka T, Okano T and Onuki T: Functional closure of visceral pleural defects by autologous tissue engineered cell sheets. Eur J Cardiothorac Surg 34: 864-869, 2008.

25. Asakawa N, Shimizu T, Tsuda Y, Sekiya S, Sasagawa T, Yamato M, Fukai F and Okano T: Pre-vascularization of in vitro three-dimensional tissues created by cell sheet engineering. Biomaterials 31: 3903-3909, 2010.

26. Shimizu T, Yamato M, Kikuchi A and Okano T: Cell sheet engineering for myocardial tissue reconstruction. Biomaterials 24: 2309-2316, 2003.

27. Shimizu T, Sekine H, Yang J, Isoi Y, Yamato M, Kikuchi A, Kobayashi E and Okano T: Polysurgery of cell sheet grafts overcomes diffusion limits to produce thick, vascularized myocardial tissues. FASEB J 20: 708-710, 2006.

28. Miyagawa S, Sawa Y, Sakakida S, Taketani S, Kondoh H, Memon IA, Imanishi Y, Shimizu T, Okano T and Matsuda H: Tissue cardiomyoplasty using bioengineered contractile cardiomyocyte sheets to repair damaged myocardium: Their integration with recipient myocardium. Transplantation 80: 1586-1595, 2005.

29. Tsumanuma Y, Iwata T, Washio K, Yoshida T, Yamada A, Takagi R, Ohno T,Lin K, Yamato M,Ishikawa I, et al: Comparison of different tissue-derived stem cell sheets for periodontal regeneration in a canine 1-wall defect model. Biomaterials 32: 5819-5825, 2011.

30. Miyahara Y, Nagaya N, Kataoka M, Yanagawa B, Tanaka K, Hao $\mathrm{H}$, Ishino $\mathrm{K}$, Ishida $\mathrm{H}$, Shimizu $\mathrm{T}$, Kangawa $\mathrm{K}$, et al: Monolayered mesenchymal stem cells repair scarred myocardium after myocardial infarction. Nat Med 12: 459-465, 2006. 
31. Okura H, Matsuyama A, Lee CM, Saga A, Kakuta-Yamamoto A Nagao A, Sougawa N, Sekiya N, Takekita K, Shudo Y, et al: Cardiomyoblast-like cells differentiated from human adipose tissue-derived mesenchymal stem cells improve left ventricular dysfunction and survival in a rat myocardial infarction model Tissue Eng Part C Methods 16: 417-425, 2010.

32. Qi Y, Du Y, Li W, Dai X, Zhao T and Yan W: Cartilage repair using mesenchymal stem cell (MSC) sheet and MSCs-loaded bilayer PLGA scaffold in a rabbit model. Knee Surg Sports Traumatol Arthrosc 22: 1424-1433, 2014.

33. Qi Y and Yan W: Mesenchymal stem cell sheet encapsulated cartilage debris provides great potential for cartilage defects repair in osteoarthritis. Med Hypotheses 79: 420-421, 2012.

34. Dumas A, Moreau MF, Ghérardi RK, Baslé MF and Chappard D: Bone grafts cultured with bone marrow stromal cells for the repair of critical bone defects: An experimental study in mice. J Biomed Mater Res A 90: 1218-1229, 2009.

35. Yang J, Yamato M, Kohno C, Nishimoto A, Sekine H, Fukai F and Okano T: Cell sheet engineering: Recreating tissues without biodegradable scaffolds. Biomaterials 26: 6415-6422, 2005.

36. Ouyang HW, Cao T, Zou XH, Heng BC, Wang LL, Song XH and Huang HF: Mesenchymal stem cell sheets revitalize nonviable dense grafts: Implications for repair of large-bone and tendon defects. Transplantation 82: 170-174, 2006.

37. Gao Z, Chen F, Zhang J, He L, Cheng X, Ma Q and Mao T: Vitalisation of tubular coral scaffolds with cell sheets for regeneration of long bones: A preliminary study in nude mice. Br J Oral Maxillofac Surg 47: 116-122, 2009.

38. Zhou Y, Chen F, Ho ST, Woodruff MA, Lim TM and Hutmacher DW: Combined marrow stromal cell-sheet techniques and high-strength biodegradable composite scaffolds for engineered functional bone grafts. Biomaterials 28: 814-824, 2007.

39. Zou XH, Cai HX, Yin Z, Chen X, Jiang YZ, Hu H and Ouyang HW: A novel strategy incorporated the power of mesenchymal stem cells to allografts for segmental bone tissue engineering. Cell Transplant 18: 433-441, 2009.

40. Qi Y, Wang Y, Yan W, Li H, Shi Z and Pan Z: Combined mesenchymal stem cell sheets and rhBMP-2-releasing calcium sulfate-rhBMP-2 scaffolds for segmental bone tissue engineering. Cell Transplant 21: 693-705, 2012.

41. Qi Y, Zhao T, Yan W, Xu K, Shi Z and Wang J: Mesenchymal stem cell sheet transplantation combined with locally released simvastatin enhances bone formation in a rat tibia osteotomy model. Cytotherapy 15: 44-56, 2013

42. Nakamura A, Akahane M, Shigematsu H, Tadokoro M, Morita Y, Ohgushi H, Dohi Y, Imamura T and Tanaka Y: Cell sheet transplantation of cultured mesenchymal stem cells enhances bone formation in a rat nonunion model. Bone 46: 418-424, 2010.

43. Ma D, Ren L, Chen F, Liu Y, Zhang J, Xue Z and Mao T: Reconstruction of rabbit critical-size calvarial defects using autologous bone marrow stromal cell sheets. Ann Plast Surg 65: $259-265,2010$

44. Zhou W, Han C, Song Y, Yan X, Li D, Chai Z, Feng Z, Dong Y, Li L, Xie X, et al: The performance of bone marrow mesenchymal stem cell - implant complexes prepared by cell sheet engineering techniques. Biomaterials 31: 3212-3221, 2010.

45. Yu M, Zhou W, Song Y, Yu F, Li D, Na S, Zou G, Zhai M and Xie C: Development of mesenchymal stem cell-implant complexes by cultured cells sheet enhances osseointegration in type 2 diabetic rat model. Bone 49: 387-394, 2011.
46. Mifune Y, Matsumoto T, Takayama K, Terada S, Sekiya N, Kuroda R, Kurosaka M, Fu FH and Huard J: Tendon graft revitalization using adult anterior cruciate ligament (ACL)-derived CD $34^{+}$cell sheets for ACL reconstruction. Biomaterials 34: 5476-5487, 2013.

47. Lui PP, Wong OT and Lee YW: Application of tendon-derived stem cell sheet for the promotion of graft healing in anterior cruciate ligament reconstruction. Am J Sports Med 42: 681-689, 2014.

48. Chang $\mathrm{CH}$, Chen $\mathrm{CH}$, Liu HW, Whu SW, Chen SH, Tsai CL and Hsiue GH: Bioengineered periosteal progenitor cell sheets to enhance tendon-bone healing in a bone tunnel. Biomed J 35: 473-480, 2012

49. Sekiya N, Tobita K, Beckman S, Okada M, Gharaibeh B, Sawa Y, Kormos RL and Huard J: Muscle-derived stem cell sheets support pump function and prevent cardiac arrhythmias in a model of chronic myocardial infarction. Mol Ther 21: 662-669, 2013

50. Kamata S, Miyagawa S, Fukushima S, Nakatani S, Kawamoto A, Saito A, Harada A, Shimizu T, Daimon T, Okano T, et al: Improvement of cardiac stem cell-Sheet therapy for chronic ischemic injury by adding endothelial progenitor cell transplantation: Analysis of layer-specific regional cardiac function. Cell Transplant 23: 1305-1319, 2014.

51. Matsuura K, Masuda S, Haraguchi Y, Yasuda N, Shimizu T, Hagiwara N, Zandstra PW and Okano T: Creation of mouse embryonic stem cell-derived cardiac cell sheets. Biomaterials 32: 7355-7362, 2011

52. Zhang W, Yang W, Liu X, Zhang L, Huang W and Zhang Y: Rapidly constructed scaffold-free embryonic stem cell sheets for ocular surface reconstruction. Scanning 36: 286-292, 2014.

53. Wakitani S, Takaoka K, Hattori T, Miyazawa N, Iwanaga T, Takeda S, Watanabe TK and Tanigami A: Embryonic stem cells injected into the mouse knee joint form teratomas and subsequently destroy the joint. Rheumatology (Oxford) 42: 162-165, 2003.

54. Kito T, Shibata R, Ishii M, Suzuki H, Himeno T, Kataoka Y, Yamamura Y, Yamamoto T, Nishio N, Ito S, et al: iPS cell sheets created by a novel magnetite tissue engineering method for reparative angiogenesis. Sci Rep 3: 1418, 2013.

55. Kawamura M, Miyagawa S, Miki K, Saito A, Fukushima S, Higuchi T, Kawamura T, Kuratani T, Daimon T, Shimizu T, et al: Feasibility, safety, and therapeutic efficacy of human induced pluripotent stem cell-derived cardiomyocyte sheets in a porcine ischemic cardiomyopathy model. Circulation 126 (Suppl 1): S29-S37, 2012.

56. Kawamura M, Miyagawa S, Fukushima S, Saito A, Miki K, Ito E, Sougawa N, Kawamura T, Daimon T, Shimizu T, et al: Enhanced survival of transplanted human induced pluripotent stem cell-derived cardiomyocytes by the combination of cell sheets with the pedicled omental flap technique in a porcine heart. Circulation 128 (Suppl 1): S87-S94, 2013.

57. Hibino N, Duncan DR, Nalbandian A, Yi T, Qyang Y, Shinoka T and Breuer CK: Evaluation of the use of an induced puripotent stem cell sheet for the construction of tissue-engineered vascular grafts. J Thorac Cardiovasc Surg 143: 696-703, 2012.

58. Tamama K, Kawasaki H, Kerpedjieva SS, Guan J, Ganju RK and Sen CK: Differential roles of hypoxia inducible factor subunits in multipotential stromal cells under hypoxic condition. J Cell Biochem 112: 804-817, 2011. 